\title{
Pattern Overlap and High Resolution Electron Backscatter Diffraction
}

\author{
Vivian Tong ${ }^{1}$, Jun Jiang ${ }^{1}$, Angus Wilkinson ${ }^{2}$ and Ben Britton ${ }^{1}$ \\ 1. Department of Materials, Imperial College London, Prince Consort Road, London, SW7 2AZ, UK \\ 2. Department of Materials. University of Oxford, Parks Road, Oxford, OX1 3PH, UK
}

High resolution electron backscatter diffraction (HR-EBSD) is a technique that can routinely measure residual elastic strain and lattice rotations with very high precision ( $10^{-4}$ in strain and rotation) [1-2] across large areas in a scanning electron microscope. The technique involves cross correlation of two or more diffraction patterns to measure subtle (sub pixel) image shifts and relate these to the relative strain and lattice rotation difference between the two patterns.

Often interfaces are of significant interest in understanding the limiting performance in real component design and understanding residual elastic strain and lattice rotation can aid the generation of new materials and microstructures. However understanding the behavior of interfaces can be difficult, as in these regions the diffraction pattern generated in the SEM may contain information from the grains either side of the interface. This may limit the precision and spatial resolution of HR-EBSD.

To explore limitations on the sensitivity of the HR-EBSD technique in the vicinity of grain boundaries and interfaces, we have performed a series of experiments using real and simulated pattern mixing, as well as some statistical analysis of the frequency of these regions that are likely to contain suspect data.

EBSD patterns were captured from well-polished annealed Zircaloy-4 using a Zeiss Auriga CrossBeam instrument in high current mode with a large aperture $(120 \mu \mathrm{m})$ combined with a Bruker eFlash $^{\mathrm{HR}}$ camera. The interaction volume of the electron beam with the sample was measured using a cross correlation method based upon the work of Chen et al [3]. The peak height measurements were compared with a simple simulation of the interaction volume, where the volume was assumed to be a half sphere crossing a vertical grain boundary of radius $\sim 60 \mathrm{~nm}$ (Figure 1 ).

With knowledge of the interaction volume, simulations of pattern mixing were performed. Intensities from the two separate grains were mixed artificially (Figure 2) to explore the influence of a less dominant pattern on the cross correlation results. Pattern mixing fractions were related to distance from the grain boundary for our measured interaction volume. It was found that the accuracy, measured with an imposed pattern shift is only significantly $\left(>2 \times 10^{-4}\right)$ affected when the interaction volume was $\sim 18 \mathrm{~nm}$ from the boundary.

The likelihood of suspect data was evaluated through consideration of the EBSD sampling strategy and the interaction volume. In typical experiments, EBSD maps are generated using a discrete sampling of the microstructure in a grid and grain boundaries are typically curved and their exact position is rarely known. Therefore the frequency of sampling close to a grain boundary was explored through sampling of a simulated Voronoi microstructure (Figure 3). The probability of sampling near a grain boundary was found to be linearly dependent on the threshold distance and inversely proportional to the step size. Therefore the probability of points in the neighborhood of a grain boundary (i.e. 1 step either side of the boundary) can be estimated. With a step size of $0.2 \mu \mathrm{m}, 30 \%$ of points in this region likely to show some overlap (i.e. the pattern contains diffraction information from both grains) and only $9 \%$ points in this neighborhood are likely to show significant strain error $\left(>2 \times 10^{-4}\right)$. 

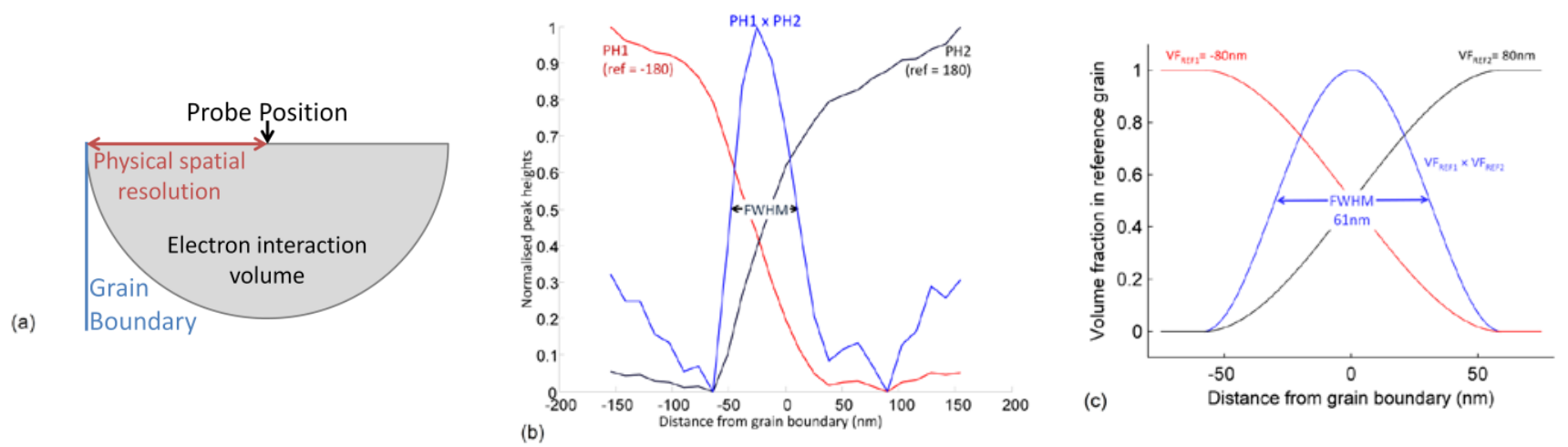

Figure 1: Measurement of the electron beam-sample interaction volume through cross correlation of diffraction patterns. (a) schematic of the half-sphere model; (b) experimetal measurement of the volume; (c) verification using a half sphere model simulation.

Pattern A

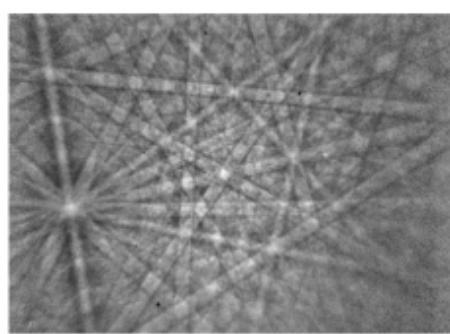

Mixed Pattern

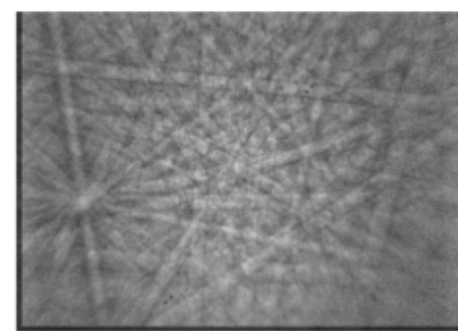

Pattern B

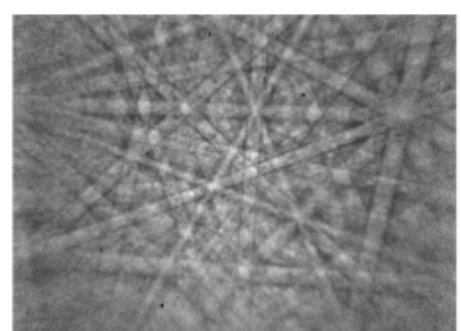

Figure 2: Experimentally obtained patterns from grains of Zircaloy-4 and the result of pattern mixing, experimentally found with overlap of the grain boundary with the interaction volume.
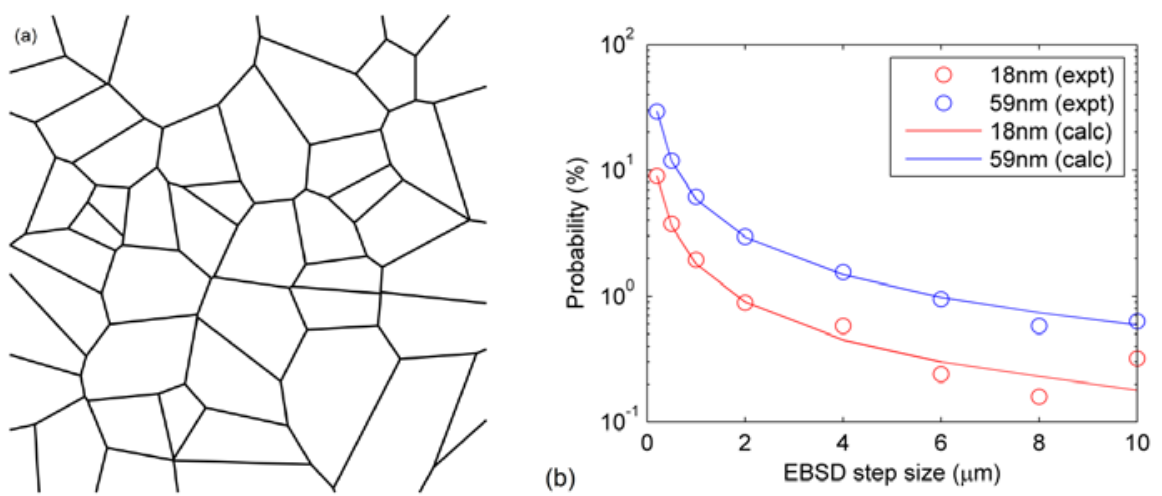

Figure 3: (a) Simulation of an example microstructure and (b) measurement of the likelihood of a point near a grain boundary, i.e. within one step size, being within a specific distance of the exact grain boundary position.

\section{References:}

[1] A.J. Wilkinson, G. Meaden, and D.J. Dingley, Ultramicroscopy 22 (2006) p. 1271

[2] T.B. Britton, J. Jiang, P.S. Karamched and A.J. Wilkinson, JOM 65 (2013) p. 1245

[3] D. Chen, J-C. Kuo and W-T. Wu, Ultramicroscopy 111 (2011) p. 1488

[4] Acknowledgements: The authors would like to thank Drs Stuart Wright, Eleanor Clarke, Phani Karamched and Philip Littlewood for helpful discussions over the years concerning pattern overlap at grain boundaries and its effects on HR-EBSD measurements. Funding contributions are acknowledged from ESPRC through the HexMat grant (EP/K034332/1), Rolls-Royce plc and AVIC-BIAM. 